$63^{\text {ème }}$ Congrès de la SFCO, 03024 (2015)

DOI:10.1051/sfco/20156303024

(C) Owned by the authors, published by EDP Sciences, 2015

\title{
Syndrome de Ascher traité par laser : un cas
}

\author{
Masson Regnault E, Catros S, Fricain J-C \\ Pôle odontologie et santé buccale CHU Bordeaux
}

Le syndrome de Ascher (SA) a été décrit pour la première fois en 1920 par Ascher, un ophtalmologiste de Prague (Ascher 1922). Le SA est caractérisé par l'association d'une tuméfaction du versant muqueux de la lèvre supérieur réalisant une double lèvre, un blépharochalasis bilatéral et un goitre thyroïdien non toxique non nécessaire au diagnostic (Barnett 1972). Un cas de SA traité par laser est rapporté.

Un patient âgé de trente ans, sans antécédent médico chirurgical a été adressé à la consultation spécialisée de pathologie de la muqueuse buccale du CHU de Bordeaux pour une tuméfaction labiale Chronique. L'interrogatoire rapportait l'apparition de cette double lèvre durant l'adolescence. Aucun antécédent familial de ce type n'était rapporté. L'aspect inesthétique de sa lèvre gênait le patient. L'examen clinique révélait en plus de la double lèvre un ptosis plus accentué du coté droit ainsi qu'un prolapsus des paupières supérieures. La palpation de la région thyroïdienne ne révélait pas de goitre. Devant ces signes cliniques, un bilan thyroïdien. La TSH était normale. Le diagnostic d'un SA a été posé. Une plastie labiale supérieure avec exérèse du tissu hypertrophique a été réalisée au laser diode à (longueur d'onde $910 \mathrm{~nm}$, puissance $3 \mathrm{~W}$ ). La plastie a été réalisée en deux temps espacés de 1 mois. Les suites opératoires ont été simples. L’analyse histopathologique était sans particularité. La blépharoplastie a été proposée au patient mais n’a pas été réalisé. Un suivi post opératoire pendant 6 mois a été instauré. Aucune récidive n’a été observée.

Des cas de double lèvres ne rentrant pas dans le cadre d'un SA ont été décrits (Parmar 2004, CostaHanemann 2004). La lèvre supérieure est le plus souvent atteinte mais des cas de la lèvre inférieure ou des deux lèvres ont été décrits (Dingman 1960). Les doubles lèvres seraient dues à une hypertrophie du chorion sans participation du muscle orbiculaire (Palma 2009). La majorité des auteurs décrit une incision elliptique associée à une plastie en Z ou W (Guerrero-santos 1967, Schwimmer 1979). Le cas présenté est le seul traité par laser. Le traitement laser offre plusieurs avantages : hémostase, absence de sutures, simplicité de l'acte.

Nom et adresse du conférencier

\section{Emmanuel MASSON REGNAULT}

CHU Bordeaux

9 rue de la Pelouse de Douet

33000 Bordeaux (France)

emmanuelmr@hotmail.fr

This is an Open Access article distributed under the terms of the Creative Commons Attribution License 4.0, which permits unrestricted use, distribution, and reproduction in any medium, provided the original work is properly cited. 